(c) American Dairy Science Association, 2003.

\title{
Effects of Including a Quantitative Trait Locus in Selection Under Different Waiting Plans of Young Bulls
}

\author{
Gamal Abdel-Azim and A. E. Freeman \\ Department of Animal Science, \\ lowa State University, Ames 50011
}

\begin{abstract}
The advantage of using the genotype of a quantitative trait locus (QTL) in selection schemes of dairy cattle was quantified using stochastic simulation. Three selection plans were studied. In the first plan, young bulls waited for $3 \mathrm{yr}$ until their sisters completed a lactation and then were evaluated and selected based on an animal model. In a second plan, young bulls waited for 5 yr until their daughters completed a lactation. An intermediate 4-yr waiting plan was also studied. Simulation was for 16 yr with overlapping generations. Population and model parameters were proportional to the U.S. Holstein population. The advantage of using a QTL was quantified as the percentage of superiority of QTL-assisted over QTLfree selection using cumulative genetic response. Percentage of superiority was reported for four selection pathways: active sires, young bulls, bull dams, and first lactation cows. A general trend was observed: low superiority in early years of selection that increased to a plateau in later years and then decreased. The superiority of the QTL information was greatest in the 3-yr waiting plan and least in the 4-yr waiting plan. Superiority at plateau for selection pathways ranged from 16 to $26 \%$ for the 3-yr waiting plan, from 3 to $12 \%$ for the $4-\mathrm{yr}$ waiting plan, and from 5 to $13 \%$ for the 5 -yr waiting plan. The contribution to selection response attributed to the QTL and the polygenes was quantified. The rate at which the favorable allele approached fixation and the accuracy of predicting breeding values on the percentage of superiority were studied.
\end{abstract}

(Key words: dairy cattle breeding, marker-assisted selection, quantitative trait loci)

Abbreviation key: $\mathbf{A S}=$ active sires, $\mathbf{B D}=$ bull dams, $\mathbf{C G R}=$ cumulative genetic response, $\mathbf{F L C}=$ first lacta-

Received December 23, 2001.

Accepted August 9, 2002.

Corresponding Author: Gamal Abdel-Azim; e-mail: gaazim@ iastate.edu.

${ }^{1}$ Journal Paper No. J-19635 of the Iowa Agriculture and Home Economics Experimental Station, Ames, Iowa. Project No. 3613, and supported by Hatch Act and State of Iowa funds. tion cows, QAS = QTL-assisted selection, $\mathbf{Q F S}=$ QTLfree selection, $\mathbf{Y B}=$ young bulls.

\section{INTRODUCTION}

The use of QTL information in dairy cattle selection has been evaluated by simulation (e.g., Meuwissen and van Arendonk, 1992; Ruane and Colleau, 1996; Spelman and Garrick, 1997; Spelman and Garrick, 1998; Spelman et al., 1999). Extra genetic response gained by QTLassisted selection (QAS) was limited when QTL information was used to augment conventional progeny testing programs (Meuwissen and van Arendonk, 1992; Spelman and Garrick, 1997; Spelman and Garrick, 1998).

It has been suggested that modifications to breeding systems are necessary to make QAS an effective tool for increasing genetic response (Larzul et al., 1997; Spelman and Garrick, 1997; Spelman et al., 1999). Spelman et al. (1999) studied the effect of a marker-assisted selection plan that used unproven young bulls (YB) at $1 \mathrm{yr}$ of age in the bull-to-bull pathway. The plan was compared with the conventional 5-yr waiting plan and it was found that using a percentage of unproven bulls on the bull-to-bull path improved superiority of marker-assisted selection. Spelman et al. (1999) used a deterministic model to predict asymptotic genetic gain ignoring the reduction in genetic variance from inbreeding and the change in the QTL allele frequency.

Ruane and Colleau (1996) used stochastic simulation to quantify the advantage of using complete genotype information of a highly polymorphic marker in selecting animals in a nucleus herd. Superiority found in the study ranged from $-0.4 \%$ in generation 1 for 8 sires and 16 dams selected per generation to $24.3 \%$ in generation 3 for 8 sires and 64 dams selected per generation. Generations were discrete, and the simulated population was closed, small, and simple in structure. Further, males were selected early (as soon as their sisters were recorded) without comparing this with the conventional progeny-testing scheme in which males are selected after their daughters complete a lactation.

The objective of the current study was to quantify the short-term superiority of using QTL genotype information to shorten the waiting period of YB. Special attention 
was given to genetic gain and to the contribution of selection pressure and accuracy of prediction to the superiority of QAS. The simulation employed parameters and population structure with mating plans and age groups similar the U.S. Holstein population.

\section{MATERIALS AND METHODS}

Numerous simulation studies have reported a decline in additional response from marker-assisted selection over generations as reviewed by Haley and Visscher (1998). Marker-assisted selection puts more emphasis on the QTL than conventional selection and causes an accelerated fixation of the QTL, hence, reaches the same genetic level sooner, not a higher level. Therefore, no general conclusion on the effect of marker-assisted selection on long-term results should be drawn (Haley and Visscher, 1998). The current study was designed with short-term results in mind. Simulation was for $16 \mathrm{yr}$, enough time for the QTL to approach fixation.

This study investigated the effect of shortening the waiting period of $\mathrm{YB}$, before their selection as active sires (AS), on the superiority of QAS over QTL-free selection (QFS). Three waiting plans were simulated: selecting YB at $3 \mathrm{yr}$ of age after their sisters completed a lactation (the 3-yr waiting plan), selecting young bulls at $5 \mathrm{yr}$ of age after their daughters completed a lactation (the 5yr waiting plan), and an intermediate 4 -yr waiting plan.

\section{Simulation}

The goal of the current study was to quantify the superiority of QAS in the conventional 5-yr waiting plan and to compare this with the 3 - and 4-yr waiting plans. The idea was to compare two situations: in the first, YB were evaluated after recording the trait of interest on their daughters, and in the second, YB were evaluated before recording the trait on the daughters. This was done by adding the list of AS to the list of YB, sorting based on estimated breeding values, and selecting a new set of AS every yr. Therefore, a YB became an AS only if his estimated breeding value exceeded any of the estimated breeding values of the AS. Results of the three waiting periods, namely 3,4 , and $5 \mathrm{yr}$, were obtained by separately replicating runs of the simulation program 50 times.

A linear additive simulation model for milk yield was used and included: management group, parity, breeding value, and residual effect. A management group was composed of herd, year, and season subclasses. Breeding value for milk yield was simulated as the aggregate effect of 40 loci in addition to one QTL. All loci were independent with gametic phase equilibrium in the base population. The total additive genetic variance and the trait
Table 1. Parameter values used in the simulation.

Genetic

Proportion of the genetic variance explained by the QTL: $15 \%$

Heritability: 0.3

Initial frequency of the favorable QTL allele: 0.1

Number of alleles at the QTL: 2

Number of QTL: 1

Number of polygenes: 40 with 2 alleles each

Nongenetic

Average number of daughters per $\mathrm{YB}^{1}: 60$

Number of YB: 21

Number of $\mathrm{AS}^{2}: 10$

Average number of milking cows: 10,000

Number of herds: 80

Percentage of records of parities 1 to 5: $33 \%, 26 \%, 19 \%, 14 \%, 8 \%$, respectively.

Culling percentages of cows of parities 1 to $5: 22 \%, 26 \%, 29 \%, 34 \%$, and $1 \%$, respectively.

\footnotetext{
${ }^{1}$ Young bulls.

${ }^{2}$ Active sires.

${ }^{3}$ Bull dams.
}

genotype distribution were the same for QAS and QFS at the beginning of each run. The model used to analyze the simulated data was

$$
\mathbf{y}=\mathbf{X b}+\mathbf{X}_{\mathrm{g}} \mathbf{g}+\mathbf{Z u}+\mathbf{e}
$$

where $\mathbf{X}_{\mathrm{g}}$ is an incidence matrix relating QTL genotypes to animals with observations, and $\mathbf{g}$ is a vector of genotype effects. The matrix $\mathbf{X}$ is an incidence matrix of all other fixed factors (i.e., parity, herd, year, and season, as described in Abdel-Azim and Freeman, 2002). The vector $\mathbf{b}$ is a vector of fixed effects to be estimated. The matrix $\mathbf{Z}$ is a design matrix relating animals to their corresponding polygenic effects $(\mathbf{u})$. The vector e contains random residuals specific to each individual. The simulation parameters used are listed in Table 1. The total phenotypic variance used to simulate yield records was $(913 \mathrm{~kg})^{2}$ of which $40 \%$ was attributed to fixed effects and $60 \%$ to random effects. Details about the simulation model and its components and parameters can be found in Abdel-Azim and Freeman (2002).

Cycles of selection were defined to facilitate the reporting of results. Simulation was for $16 \mathrm{yr}$ for all systems. For the conventional 5-yr waiting plan, the $16 \mathrm{yr}$ consisted of three cycles of selection as follows: in yr 6 to 10 the best of the young bulls tested in yr 1 to 5 became active, in yr 11 to 15 the best of the YB tested in yr 6 to 10 became active, and in yr 16 the best of the YB tested in yr 11 became active. Although only three cycles of selection were defined, a proven group of YB became active every year after yr 5 .

For the shorter waiting plans, more than three cycles of selection were made in $16 \mathrm{yr}$. The 3 -yr waiting plan 
comprised five cycles of selection as follows: in yr 4 to 6 the best of the YB born in yr 0 to 2 became active, then in yr 7 to 9 the best of the YB born in yr 3 to 5 became active, etc.

Although these cycles are also encountered in dairy populations selected based on progeny testing, each cycle marks a major increase in the level of cumulative genetic response (CGR). In our simulation, for example, parents were unselected in the first cycle, the second cycle contained the first selected set of parents based on estimated breeding values that were not available before, the third cycle contained the second set of selected parents whose parents were also selected, etc. This progressive increase in CGR every cycle justified the use of cycles instead of years to report results by averaging yearly results of each cycle.

\section{Examined Aspects of QAS Superiority}

For each of the three waiting plans, the simulation program was run twice: one run with the QTL as a fixed factor in the mixed model [1], and a second run with a conventional animal model in which the whole breeding value was estimated without fitting a component for the QTL. A run in the context of the current study means simulating data and performing genetic evaluation once a yr for 16 consecutive yr.

Results from each replicate included: cumulative genetic response, correlation between estimated and true breeding values, and favorable QTL allele frequency. These were obtained each year for AS, YB, bull dams (BD), and first lactation cows (FLC) in the population. First, true breeding values were averaged for each of the four animal groups to indicate cumulative annual genetic response. True breeding values were employed in computing genetic responses but selection decisions were based on estimated breeding values. Correlation coefficients were computed between true and estimated breeding values each year. The coefficients computed were taken to indicate accuracy of estimating breeding value for selection pathways. Favorable QTL allele frequency was computed within each pathway to reflect the intensity of selection pressure on the QTL.

Cumulative genetic responses were presented in terms of the percentage of superiority of animals specific to a selection pathway under QAS over corresponding animals under QFS. Mean percentage of superiority was computed as

$$
\begin{gathered}
\frac{100}{50} \sum_{i=1}^{50}\left(\left(\overline{\mathrm{BV}}_{Q A S}\right)_{i}\right. \\
\left.-\left(\overline{\mathrm{BV}}_{Q F S}\right)_{i}\right) /\left|\left(\overline{\mathrm{BV}}_{Q F S}\right)_{i}\right|,\left|\left(\overline{\mathrm{BV}}_{Q F S}\right)_{i}\right| \neq 0
\end{gathered}
$$

where $\left(\overline{\mathrm{BV}}_{Q A S}\right)_{i}$ and $\left(\overline{\mathrm{BV}}_{Q F S}\right)_{i}$ are the true breeding values for QAS and QFS schemes averaged for individuals within any of the four concerned groups, namely AS, YB, $\mathrm{BD}$, and FLC of the $i$ th replicate of 50. In early years, $\left(\overline{\mathrm{BV}}_{Q A S}\right)_{i}$ and $\left(\overline{\mathrm{BV}}_{Q F S}\right)_{i}$ may differ in sign hence division was by the absolute value of $\left(\overline{\mathrm{BV}}_{Q F S}\right)_{i}$ in formula [2].

Genetic response per year could be expressed as a function of selection intensity, $i$, correlation between true and predicted breeding values, $r$, the additive genetic standard deviation, $\sigma_{g}$, and the generation interval, $L$. Response superiority of QAS scheme over OFS scheme in cases where $L$ and $i$ are equal for both schemes, expressed as the difference in cumulative genetic response per year between QAS and QFS divided by QFS, can be written as

$$
\frac{r_{1} \sqrt{2 p_{1} q_{1} \alpha^{2}+\sigma_{g p}^{2}}}{\mathrm{r}_{2} \sqrt{2 p_{2} q_{2} \alpha^{2}+\sigma_{g p}^{2}}}-1
$$

where $\sigma_{g p}$ is the additive genetic standard deviation for the polygenic component that is assumed equal under QAS and QFS in the simulation and analysis; $\alpha$ is the average effect of QTL allele substitution; $p_{1}$ and $p_{2}$ are favorable allele frequencies under QAS and QFS, respectively; $q_{1}$ and $q_{2}$ are $\left(1-p_{1}\right)$ and $\left(1-p_{2}\right)$, respectively; and $r_{1}$ and $r_{2}$ are prediction accuracy of breeding values under QAS and QFS, respectively. The values of $\alpha$ and $\sigma_{g p}$ are constant across the years of simulation and hence have no effect on the trend of superiority across years. Expression [3] is explained in Appendix 1.

\section{RESULTS AND DISCUSSION}

\section{Total Cumulative Genetic Response Superiority}

Total cumulative genetic response (CGR) under QAS and QFS was computed each year for AS, YB, BD, and FLC (Tables 2, 3, 4, and 5). Formula [2] was used to compute the average percentage of superiority of QAS over QFS from the results of 50 replicates. Tables 2 to 5 list the percentage of superiority for the pathways across waiting plans. This is useful in assessing the benefit of QAS in the pathways and comparing this for the waiting plans studied. The trend of the percentage of superiority can be easily observed by looking at results of the 3-yr waiting plans where more selection cycles were made. The trend showed lower superiority in early years of selection that increased to a maximum and then started to decrease when the frequency of the favorable QTL allele passed 0.5, as will follow from Figure 1. The superiority of QAS then decreased as the QTL approached fixation.

Tables 2 to 5 list absolute averages of CGR in kilograms and the contribution of the QTL component to 
Table 2. Percentage of superiority of $\mathrm{QAS}^{1}$ over $\mathrm{QFS}^{2}$, cumulative genetic response (CGR), and percentage of QTL contribution to CGR (QTL\%) in active sires of three waiting plans ${ }^{3}$. Parenthetical values are standard errors.

\begin{tabular}{|c|c|c|c|c|c|c|}
\hline \multirow[b]{2}{*}{ Cycle } & \multirow[b]{2}{*}{ Years } & \multirow{2}{*}{$\begin{array}{l}\text { QAS } \\
\text { Superiority } \%\end{array}$} & \multicolumn{2}{|c|}{ QAS } & \multicolumn{2}{|c|}{ QFS } \\
\hline & & & QTL\% & $\mathrm{CGR}^{4}$ & QTL\% & CGR \\
\hline \multicolumn{7}{|c|}{ 3-Yr Waiting Plan } \\
\hline 2 & $4-6$ & $17.2(3.5)$ & 32.4 & $385.5(13.0)$ & 11.4 & 349.2 \\
\hline 3 & $7-9$ & $25.7(2.2)$ & 50.0 & $954.8(17.1)$ & 24.0 & 786.6 \\
\hline 4 & $10-12$ & $17.6(1.3)$ & 45.5 & $1322.7(14.2)$ & 28.7 & $1139.9(14.8)$ \\
\hline 5 & $13-15$ & 13.5 (1.1) & 39.3 & 1614.7 & 31.8 & 1437.7 \\
\hline & 16 & $11.3(1.7)$ & 36.1 & $1770.5(19.0)$ & 32.5 & $1603.2(22.9)$ \\
\hline \multicolumn{7}{|c|}{ 4-Yr Waiting Plan } \\
\hline 2 & $5-8$ & $2.2(0.4)$ & 18.5 & 511.5 & 16.5 & 502.0 \\
\hline 3 & $9-12$ & $5.4(0.8)$ & 37.3 & $1118.2(14.0)$ & 26.5 & $1072.3(15.2)$ \\
\hline 4 & $13-16$ & $5.4(0.8)$ & 37.1 & 1570.6 & 27.5 & $1502.8(15.6)$ \\
\hline \multicolumn{7}{|c|}{ 5-Yr Waiting Plan } \\
\hline 2 & $6-10$ & $1.8(0.3)$ & 19.1 & $543.1(10.1)$ & 18.3 & $533.7(9.8)$ \\
\hline 3 & $11-15$ & $7.0(0.7)$ & 35.4 & $1196.2(12.8)$ & 26.7 & 1125.9 \\
\hline & 16 & $6.0(1.4)$ & 36.2 & $1472.6(21.3)$ & 28.2 & $1398.0(23.3)$ \\
\hline
\end{tabular}

${ }^{1}$ QTL-assisted selection.

${ }^{2} \mathrm{QTL}-$ free selection.

${ }^{3}$ Young bulls waited 3,4 , and 5 yr before becoming active.

${ }^{4}$ Additive genetic variance $=\mathrm{h}^{2}(0.6)(913 \mathrm{~kg})^{2}$.

the CGR as a percentage. For example, under the 3-yr waiting plan of the QAS scheme CGR for AS reached $1770.5 \mathrm{~kg}$ in yr 16 with a standard error of $19 \mathrm{~kg}$ (Table 2). The contribution of the QTL component was $36.1 \%$ of the $1770.5 \mathrm{~kg}$ or $639.2 \mathrm{~kg}$ (i.e., $1131.3 \mathrm{~kg}$ was the contribution of the polygenes in the final year).

The QTL response superiority of QAS over QFS was computed as the difference between QAS and QFS aver-

Table 3. Percentage of superiority of $\mathrm{QAS}^{1}$ over $\mathrm{QFS}^{2}$, cumulative genetic response (CGR), and percentage of QTL contribution to CGR (QTL\%) in young bulls of three waiting plans ${ }^{3}$. Parenthetical values are standard errors.

\begin{tabular}{|c|c|c|c|c|c|c|}
\hline \multirow[b]{2}{*}{ Cycle } & \multirow[b]{2}{*}{ Years } & \multirow{2}{*}{$\begin{array}{l}\text { QAS } \\
\text { Superiority \% }\end{array}$} & \multicolumn{2}{|c|}{ QAS } & \multicolumn{2}{|c|}{ QFS } \\
\hline & & & QTL\% & $\mathrm{CGR}^{4}$ & QTL\% & CGR \\
\hline \multicolumn{7}{|c|}{ 3-Yr Waiting $\operatorname{Plan}^{5}$} \\
\hline $\begin{array}{l}3 \\
4 \\
5\end{array}$ & $\begin{array}{l}7-9 \\
10-12 \\
13-15 \\
16\end{array}$ & $\begin{array}{l}11.5(1.9) \\
20.4(1.6) \\
16.9(1.3) \\
13.4(1.8)\end{array}$ & $\begin{array}{l}46.2 \\
51.8 \\
46.0 \\
41.1\end{array}$ & $\begin{array}{rr}565.0 & (15.1) \\
989.9 & (15.7) \\
1329.3 & (12.8) \\
1537.7 & (18.6)\end{array}$ & $\begin{array}{l}16.7 \\
24.0 \\
29.2 \\
31.7\end{array}$ & $\begin{array}{rr}514.6 & (13.8) \\
836.8 & (14.9) \\
1152.8 & (14.4) \\
1366.4 & (19.5)\end{array}$ \\
\hline \multicolumn{7}{|c|}{ 4-Yr Waiting Plan } \\
\hline $\begin{array}{l}3 \\
4\end{array}$ & $\begin{array}{l}9-12 \\
13-16\end{array}$ & $\begin{array}{l}7.1(1.2) \\
5.8(1.0)\end{array}$ & $\begin{array}{l}39.2 \\
42.2\end{array}$ & $\begin{array}{r}757.5(11.7) \\
1203.2(14.5)\end{array}$ & $\begin{array}{l}22.4 \\
27.1\end{array}$ & $\begin{array}{r}718.0(11.6) \\
1148.9(14.8)\end{array}$ \\
\hline \multicolumn{7}{|c|}{ 5-Yr Waiting Plan } \\
\hline 3 & $\begin{array}{l}11-15 \\
16\end{array}$ & $\begin{array}{ll}8.1 & (0.9) \\
9.0 & (1.6)\end{array}$ & $\begin{array}{l}38.5 \\
41.5\end{array}$ & $\begin{aligned} 883.4 & (12.2) \\
1218.0 & (22.5)\end{aligned}$ & $\begin{array}{l}24.7 \\
27.6\end{array}$ & $\begin{aligned} 826.0 & (11.8) \\
1125.5 & (23.8)\end{aligned}$ \\
\hline
\end{tabular}

${ }^{1}$ QTL-assisted selection.

${ }^{2}$ QTL-free selection.

${ }^{3}$ Young bulls waited 3, 4, and 5 yr before becoming active.

${ }^{4}$ Additive genetic variance $=\mathrm{h}^{2}(0.6)(913 \mathrm{~kg})^{2}$.

${ }^{5}$ Results of previous years were not reported because of the similarity of QAS and QFS responses in these years. 
Table 4. Percentage of superiority of $\mathrm{QAS}^{1}$ over $\mathrm{QFS}^{2}$, cumulative genetic response (CGR), and percentage of QTL contribution to CGR (QTL\%) in bull dams of three waiting plans ${ }^{3}$. Parenthetical values are standard errors.

\begin{tabular}{|c|c|c|c|c|c|c|}
\hline \multirow[b]{2}{*}{ Cycle } & \multirow[b]{2}{*}{ Years } & \multirow{2}{*}{$\begin{array}{l}\text { QAS } \\
\text { Superiority } \%\end{array}$} & \multicolumn{2}{|c|}{ QAS } & \multicolumn{2}{|c|}{ QFS } \\
\hline & & & QTL\% & $\mathrm{CGR}^{4}$ & QTL $\%$ & CGR \\
\hline \multicolumn{7}{|c|}{ 3-Yr Waiting Plan } \\
\hline 2 & $4-6$ & $16.7(1.2)$ & 55.2 & $816.4(11.6)$ & 21.1 & $710.5(12.1)$ \\
\hline 3 & $7-9$ & $14.9(1.0)$ & 50.9 & 1008.7 & 25.4 & 923.3 \\
\hline 4 & $10-12$ & 16.7 & 45.8 & 1350.6 & 28.1 & 1169.9 \\
\hline 5 & $13-15$ & $11.1(0.8)$ & 39.3 & 1624.5 & 30.8 & 1471.0 \\
\hline & 16 & $8.6(1.3)$ & 35.5 & $1800.6(16.2)$ & 31.1 & $1666.3(20.2)$ \\
\hline \multicolumn{7}{|c|}{ 4-Yr Waiting Plan } \\
\hline 2 & $5-8$ & $12.1(0.9)$ & 50.7 & $897.5(9.8)$ & 21.8 & $807.7(9.7)$ \\
\hline 3 & $9-12$ & $10.9(0.8)$ & 47.1 & 1192.0 & 25.9 & 1083.7 \\
\hline 4 & $13-16$ & $8.3(0.7)$ & 39.6 & $1595.5(12.0)$ & 28.5 & $1485.3(14.1)$ \\
\hline \multicolumn{7}{|c|}{ 5-Yr Waiting Plan } \\
\hline 2 & $6-10$ & $10.7(0.7)$ & 47.7 & $947.5(9.3)$ & 23.9 & $858.2(8.0)$ \\
\hline 3 & $11-15$ & $13.1(0.6)$ & 45.1 & 1312.4 (11.8) & 27.6 & 1167.2 \\
\hline & 16 & $9.2(1.4)$ & 39.6 & 1589.8 & 29.9 & $1466.4(22.1)$ \\
\hline
\end{tabular}

${ }^{1} \mathrm{QTL}$-assisted selection.

${ }^{2}$ QTL-free selection.

${ }^{3}$ Young bulls waited 3,4 , and 5 yr before becoming active.

${ }^{4}$ Additive genetic variance $=\mathrm{h}^{2}(0.6)(913 \mathrm{~kg})^{2}$.

ages. Figure 1 shows the relationship between QTL response superiority and the QTL favorable allele frequency in FLC of the 3-yr waiting plan. The frequency shown in the graph is an average of the levels of the favorable allele in the two systems, namely QAS and QFS. Figure 1 shows a decline in the rate of increase of QTL response superiority after the favorable QTL allele passed the 0.5 frequency. The rate of increase then be-

Table 5. Percentage of superiority of $\mathrm{QAS}^{1}$ over $\mathrm{QFS}^{2}$, cumulative genetic response (CGR), and percentage of QTL contribution to CGR (QTL\%) in first-lactation cows of three waiting plans ${ }^{3}$. Parenthetical values are standard errors.

\begin{tabular}{|c|c|c|c|c|c|c|}
\hline \multirow[b]{2}{*}{ Cycle } & \multirow[b]{2}{*}{ Years } & \multirow{2}{*}{$\begin{array}{l}\text { QAS } \\
\text { Superiority } \%\end{array}$} & \multicolumn{2}{|c|}{ QAS } & \multicolumn{2}{|c|}{ QFS } \\
\hline & & & QTL\% & $\mathrm{CGR}^{4}$ & QTL\% & CGR \\
\hline \multicolumn{7}{|c|}{ 3-Yr Waiting Plan } \\
\hline 2 & $4-6$ & $4.6(5.8)$ & 42.0 & $39.3(6.9)$ & 98.9 & $36.2(6.6)$ \\
\hline 3 & $7-9$ & $16.9(3.3)$ & 42.6 & 312.1 & 14.8 & $278.1(10.4)$ \\
\hline 4 & $10-12$ & $20.9(1.7)$ & 47.6 & $674.1(12.1)$ & 24.3 & $571.3(13.6)$ \\
\hline 5 & $13-15$ & $15.7(1.0)$ & 44.6 & 1017.9 & 28.9 & $888.5(12.2)$ \\
\hline & 16 & $13.6(1.5)$ & 41.6 & 1232.4 (15.5) & 30.9 & $1092.2(17.8)$ \\
\hline \multicolumn{7}{|c|}{ 4-Yr Waiting Plan } \\
\hline 2 & $5-8$ & $2.6(1.9)$ & 22.9 & $103.6(9.7)$ & 18.1 & $102.3(9.7)$ \\
\hline 3 & $9-12$ & $2.1(0.7)$ & 29.8 & $481.2(12.5)$ & 22.8 & $474.3(12.6)$ \\
\hline 4 & $13-16$ & $2.9(0.6)$ & 36.9 & $947.1(12.8)$ & 27.6 & $927.0(13.6)$ \\
\hline \multicolumn{7}{|c|}{ 5-Yr Waiting Plan } \\
\hline 2 & $6-10$ & $-1.2(2.3)$ & 26.9 & $151.9(10.1)$ & 27.2 & $152.9(10.2)$ \\
\hline 3 & $11-15$ & $2.7(0.5)$ & 30.4 & 607.0 & 24.6 & $591.8(12.6)$ \\
\hline & 16 & $4.8(1.2)$ & 35.2 & $933.4(16.6)$ & 27.3 & 896.4 \\
\hline
\end{tabular}

${ }^{1}$ QTL-assisted selection.

${ }^{2}$ QTL-free selection.

${ }^{3}$ Young bulls waited 3, 4, and 5 yr before becoming active.

${ }^{4}$ Additive genetic variance $=\mathrm{h}^{2}(0.6)(913 \mathrm{~kg})^{2}$. 


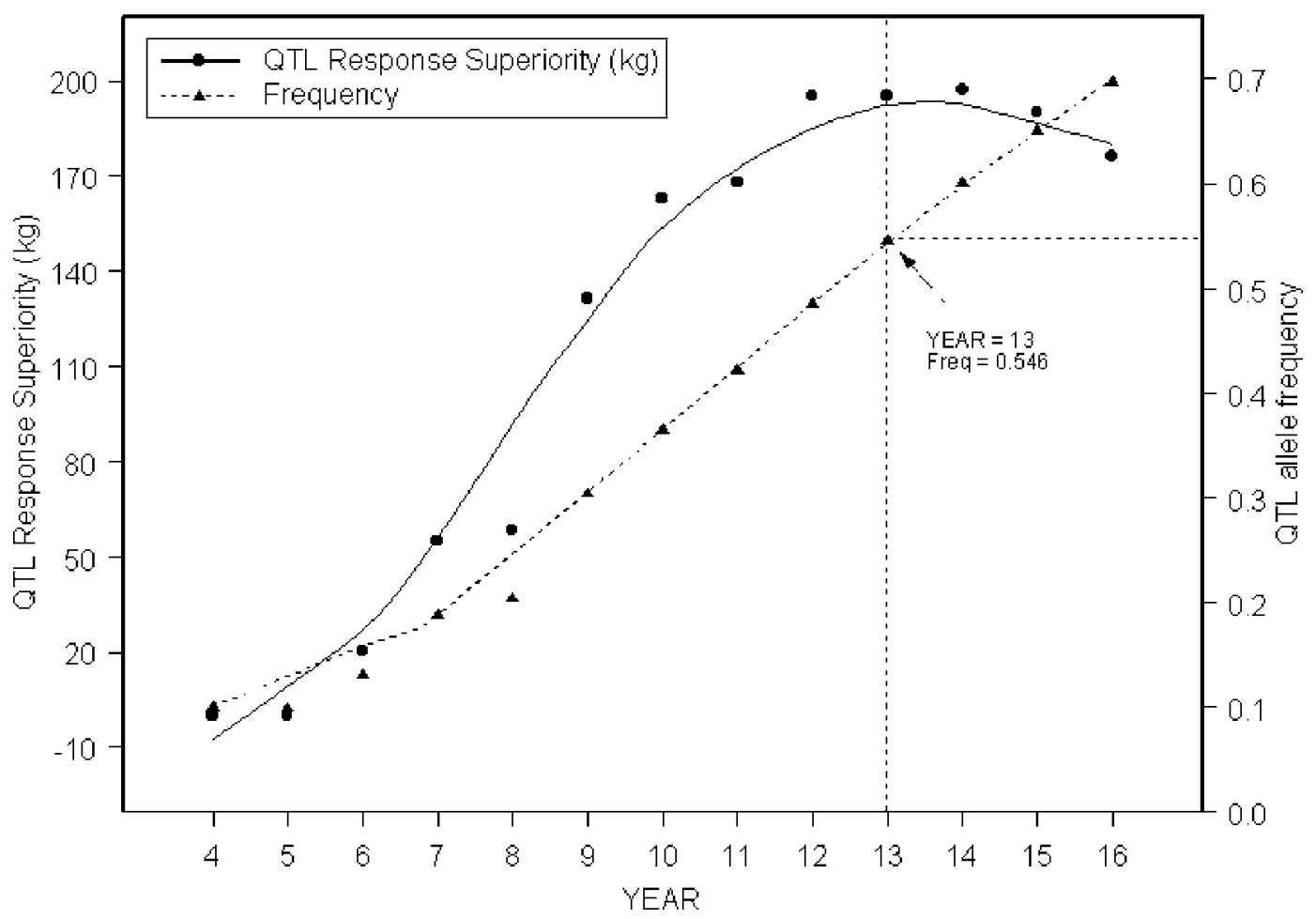

Figure 1. The relationship between superiority of QTL-assisted selection over QTL-free selection (left Y-axis) and the change in the QTL frequency (right Y-axis). Graph presents the relationship in FLC under the 3-yr waiting plan. Superiority is computed based on the component of the cumulative genetic response contributed by the QTL.

came 0 , and the superiority eroded after that. At yr 13, frequency was 0.546 , which is the closest data point to 0.5 frequency. Notice the slowing rate of increase in QTL superiority around the marked point that resides in the neighborhood of the 0.5 frequency and coincides with the plateau of QTL superiority.

The superiority under the 3-yr waiting plan was distinctively higher than the 4- and 5-yr waiting plans for all pathways. The QAS percentage of superiority of YB ranged from 11.5 to $20.4 \%$ under the 3 -yr waiting plan compared with 5.8 to $7.1 \%$ and 8.1 to $9 \%$ under the 4and 5-yr waiting plans, respectively (Table 3 ). This is caused by the greater number of cycles of selection completed in $16 \mathrm{yr}$ under the 3-yr waiting plan than under other plans, and it indicates the importance of using QTL information early in selection. In Tables 2 to 5, an advantage of the 5-yr waiting plan over the 4-yr waiting plan was observed for the percentage of superiority. This indicates that accuracy of evaluation more than compensated for the 1-yr increase in generation interval.

There were differences in the magnitude and trend of superiority among the four groups of animals. YB showed greater superiority than AS, particularly for the 4- and 5 -yr waiting plans because of the more effective conventional QFS with AS in such plans (Tables 2 and 3). Smith
(1967) concluded that when normal selection is less effective, selection assisted by identified loci becomes more effective. For the females, BD showed a high superiority at the beginning that gradually decreased as the QTL approached fixation (Tables 4 and 5). Selection intensity was the highest in BD among all groups studied, and hence more selection pressure was placed on the QTL in BD. FLC showed a lag in superiority behind all other groups because response in cows is not caused by directly selecting them but as a consequence of selecting their sires.

\section{Polygenic and QTL Cumulative Genetic Response Superiority}

Figures 2, 3, and 4 model trends of superiority of QAS over QFS contributed by the polygenes and QTL separately. Superiority is computed as the difference in the annual cumulative genetic response between QAS and QFS and partitioned for the aggregate genotypic effects of the polygenes and the genotypic effects of the identified QTL. Locally weighted regression smoothing was used to produce the curves.

In all waiting plans there is an opposite trends of the superiority of the polygenes and the QTL. Ruane and 


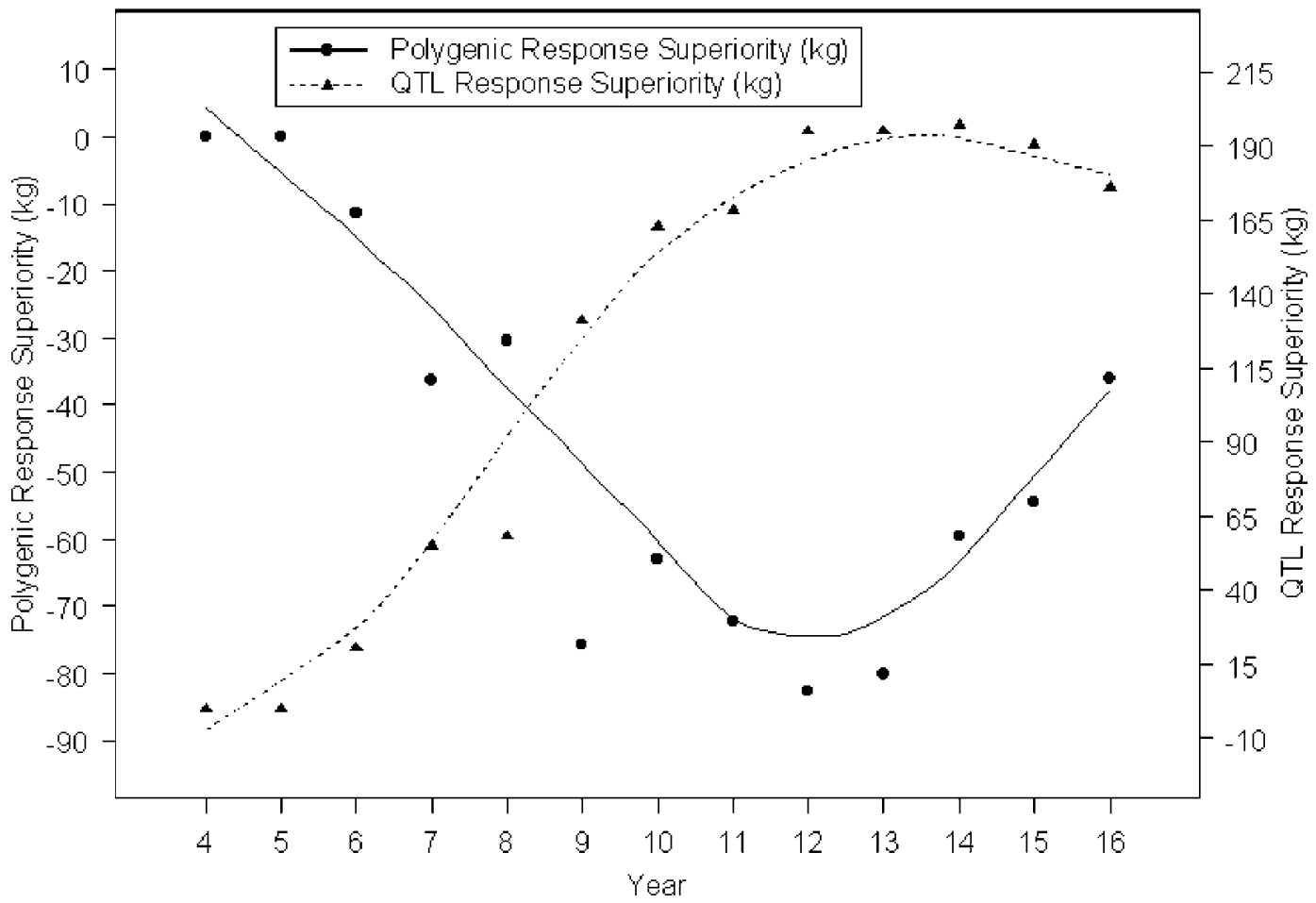

Figure 2. Response superiority of QTL-assisted selection over QTL-free selection partitioned for the polygenes (left Y-axis) and the QTL (right Y-axis). Graph presents results of FLC under the 3-yr waiting plan.

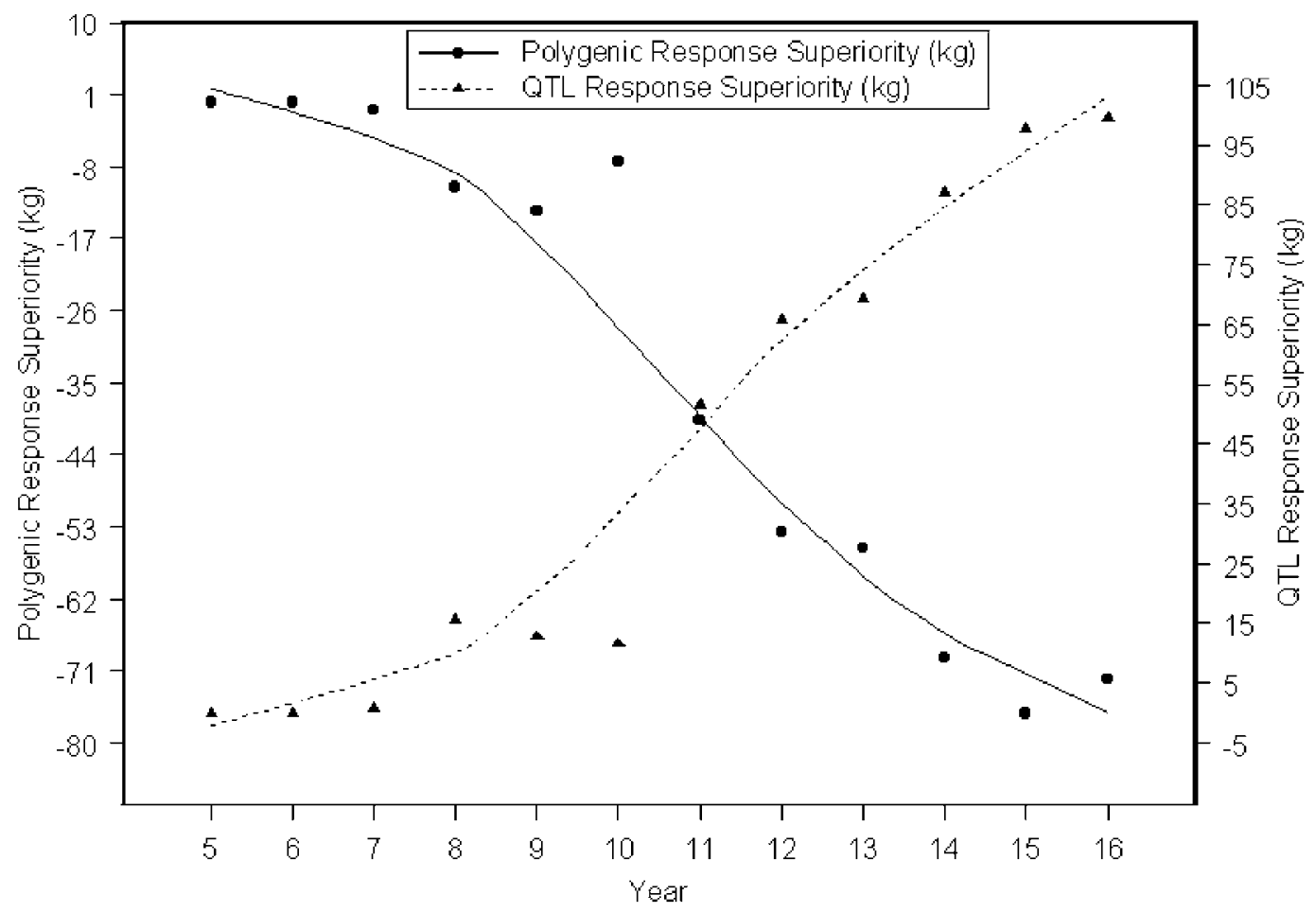

Figure 3. Response superiority of QTL-assisted selection over QTL-free selection partitioned for the polygenes (left Y-axis) and the QTL (right Y-axis). Graph presents results of FLC under the 4-yr waiting plan. 


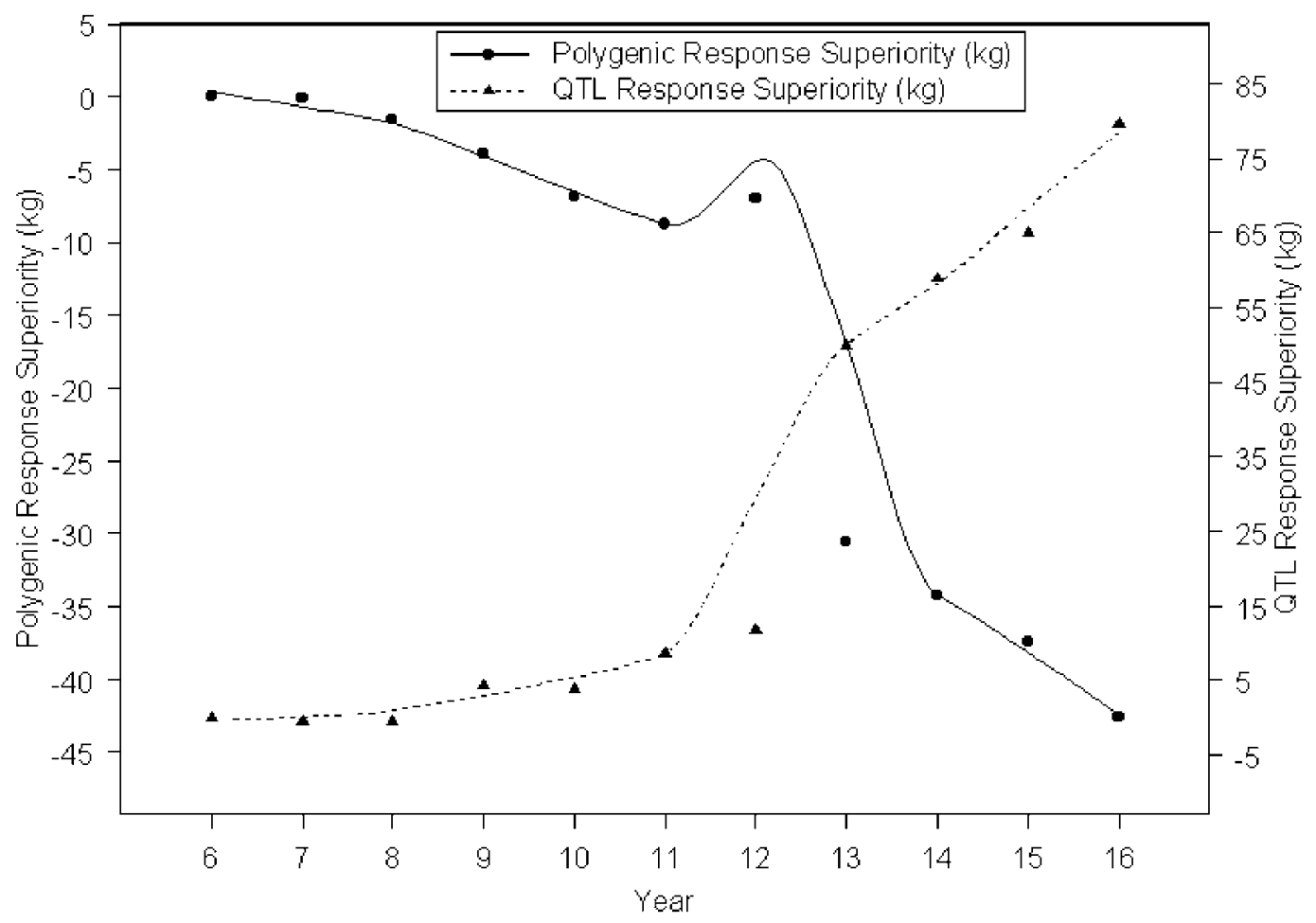

Figure 4. Response superiority of QTL-assisted selection over QTL-free selection partitioned for the polygenes (left Y-axis) and the QTL (right Y-axis). Graph presents results of FLC under the 5-yr waiting plan.

Colleau (1996) showed that marker-assisted selection increased the QTL response but tended to reduce the polygenic response. The inferiority of the polygenic response is shown by the negative left Y-axis of the polygenic response superiority.

More cycles of YB selection were made in the 3-yr waiting plan than in the 4- and 5-yr waiting plans, such that superiority trends of the QTL and the polygenes seem to be more developed under that plan (Figures 2, 3 , and 4). In Figure 2, the superiority trend curves are characterized by an increase to a convex plateau followed by a decrease for the QTL curve and by a decrease to a concave base level followed by an increase for the polygenes curve. In Figure 3, the trends are less developed, but points of the last $3 \mathrm{yr}$ hint at a near plateau for the QTL superiority and an upward gain for the polygenic superiority. The development of the superiority trend is the least in the 5-yr waiting plan because it had fewest cycles of YB selection. If the time before the intersection of the two superiority curves of the QTL and the polygenes indicates the speed of the trend development, the intersection should occur between yr 8 and 9 for the 3yr waiting plan, in yr 11 for the 4 -yr waiting plan, and in yr 13 for the 5-yr waiting plan (Figures 2,3 , and 4). This indicates that the advantage seen in the 3 -yr waiting plan over other plans was mainly a result of acceler- ated fixation of the QTL, which caused an improvement in time (accelerated gain) rather than in the absolute level. This is in agreement with the analysis that Haley and Visscher (1998) made of this phenomenon in a different context.

\section{Role of Accuracy and Gene Frequency in Affecting Response Superiority}

To study the effect of the nonconstant factors (QTL gene frequency and prediction accuracy), the superiority in expression [3] was plotted in two steps for all waiting plans (Figure 5). Data used in the graph were those of the FLC because of their relatively large number compared with males and $\mathrm{BD}$, and because of their equal generation interval under QAS and QFS. First, only $\sqrt{2 p_{1} q_{1}}$ and $\sqrt{2 p_{2} q_{2}}$ were plotted against years of simulation to magnify the effect of the QTL allele frequency on the cumulative genetic response in both systems and hence on the superiority of QAS over QFS (left column, Figure 5). The expected convex trend is most clear for QAS under the 3-yr waiting plan (top left panel, Figure 5). The QFS curve shows a slower evolution of the QTL and consequently slower response. Second, $r_{1}$ and $r_{2}$ were multiplied by $\sqrt{2 p_{1} q_{1}}$ and $\sqrt{2 p_{2} q_{2}}$ as the second noncon- 


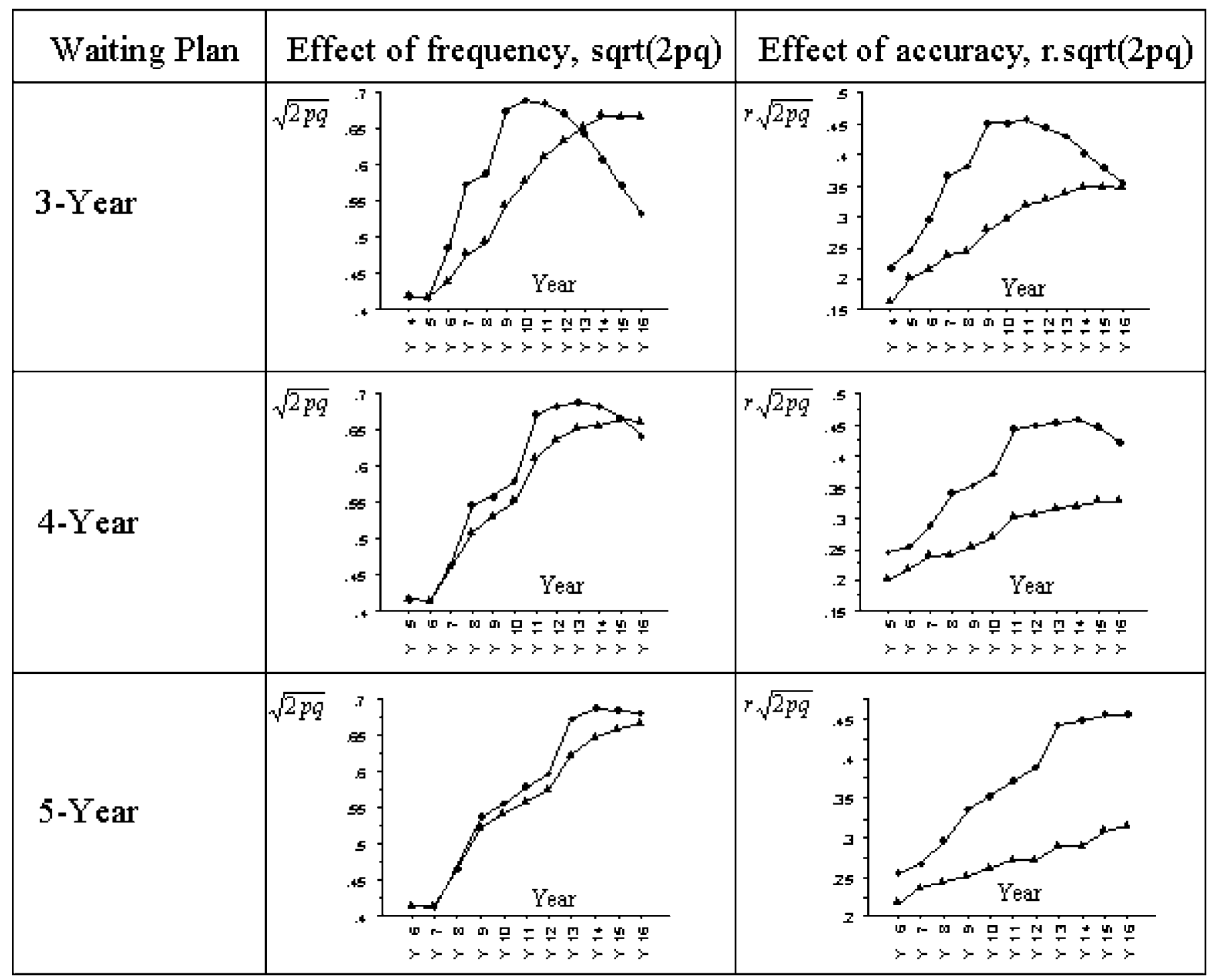

Figure 5. Factors of cumulative genetic response in FLC plotted in two steps for 3-, 4-, and 5-yr waiting plans (from top to bottom). The two curves per graph are for QTL-assisted selection (solid circle) and QTL-free selection (solid upward triangle).

stant variable in [3] to demonstrate the effect of prediction accuracy (right column, Figure 5). Step 2 (right columns, Figure 5) clearly shows the effect of the higher prediction accuracy under QAS, which preserved the QAS response advantage over QFS for more years. Notice the delayed trend development under the 4-yr and 5 -yr waiting plans (middle and bottom rows, Figure 5).

The stepwise graph of Figure 5 illustrates the effect of gene frequency on response to selection and shows the importance of accuracy on keeping QAS advantageous to QFS for a longer time. Moreover, the lag among the three waiting plans was further demonstrated in the graph. A secondary function of the stepwise graph was to validate our results by showing the consistency of gene frequency and accuracy with genetic response.

\section{CONCLUSIONS}

This study showed that the advantage of QAS is greatest when used before the daughters of YB complete their first record. When YB were used after their daughters completed a lactation record, the superiority dramatically dropped. Such waiting caused the normal selection to become more effective which in turn reduced the superiority of QAS. This is in agreement with the classic theoretical work of Smith (1967).

The study also showed no advantage to the 4-yr waiting plan. The 4-yr waiting plan involved 1 more yr of unnecessary waiting. In fact, the 5-yr waiting plan showed an advantage over the 4-yr waiting plan due to the completion of the first record on daughters of YB 
that waited for $5 \mathrm{yr}$. This clearly demonstrates the need to consider modifying the current systems of dairy cattle breeding to maximize the benefit of using identified QTL in selection.

Although superiority of QAS increased in early years of using the QTL, it eventually decreased after the favorable QTL allele passed the 0.5 frequency. This reduction in superiority is caused by the reduction in variance of the QTL as a result of the accelerated increase of QTL allele frequency when it passes the 0.5 frequency as discussed by Haley and Visscher (1998). Eventually, the advantage of using a QTL in selection diminishes as the QTL approaches fixation, consistent with Gibson's findings (1994) in an idealistic population. Using QTL in selection is not uniformly beneficial. The benefit declines to zero as the identified QTL is fixed.

The study showed differences in magnitude and trend of superiority among the four groups of animals studied here. A higher superiority in groups where conventional selection is less effective was observed, for example the better superiority in YB than AS. In addition, the group of $\mathrm{BD}$ was the earliest to benefit from the use of genomic information in selection. In contrast, FLC showed a lag in superiority behind other groups, because they were not directly selected and there was a time lag for superiority to be transmitted to the cows.

The inferiority of the polygenic response of the QAS scheme was clearly demonstrated here for all three plans while the frequency of the QTL was low. Ruane and Colleau (1996) obtained lower polygenic superiority with the higher QTL superiority of a marker-assisted selection scheme over a conventional scheme in a closed small nucleus herd. We showed a decreasing polygenic superiority trend to a concave minimum that accompanied an increasing QTL superiority trend to a convex plateau. But we also showed that the polygenic inferiority bounced back up as the QTL approached fixation, particularly with the 3 -yr waiting plan.

Finally, the study demonstrated the impact of selection pressure and prediction accuracy on the trend of cumulative genetic response for QAS and QFS schemes under the three waiting plans. The shape of the trend and the lag of the QFS scheme behind the QAS scheme were shown to be due to the gene frequency component, and the duration of the advantage of QAS was shown to be caused by its corresponding higher prediction accuracy. Factors such as generation interval and selection intensity, although affecting genetic response, did not impact superiority of QAS over QFS in FLC. The study is useful for future economic studies that target specific selection pathways and groups of animals to maximize efficiency of breeding programs. However, the results reported here assumed complete genotype information. Studies of incomplete genotype information are needed.

\section{REFERENCES}

Abdel-Azim, G., and A. E. Freeman. 2002. Superiority of QTL-assisted selection in dairy cattle breeding schemes. J. Dairy Sci. 85:1869-1880.

Gibson, J. P. 1994. Short-term gain at the expense of long-term response with selection of identified loci. Proc. 5th World Congr. Genet. Appl. Livest. Prod., Guelph, Canada 21:202-204.

Haley, C. S., and P. M. Visscher. 1998. Strategies to utilize markerquantitative trait loci associations. J. Dairy Sci. 81:85-97.

Larzul, C., E. Manfredi, and J. M. Elsen. 1997. Potential gain from including major gene information in breeding value estimation. Genet. Sel. Evol. 29:161-184.

Meuwissen, T. H. E., and J. A. M. van Arendonk. 1992. Potential improvement in rate of genetic gain from marker-assisted selection in dairy cattle breeding schemes. J. Dairy Sci. 75:1651-1659.

Meuwissen, T. H. E., and J. A. M. van Arendonk. 1996. The use of marker haplotypes in animal breeding schemes. Genet. Sel. Evol. 28:161-176.

Ruane, J., and J. J. Colleau. 1996. Marker-assisted selection for a sexlimited character in nucleus breeding population. J. Dairy Sci. 79:1666-1678.

Smith, C. 1967. Improvement of metric traits through specific genetic loci. Anim. Prod. 9:349-358.

Spelman, R. J., and D. J. Garrick. 1997. Utilisation of marker assisted selection in a commercial dairy cow population. Livestock Prod. Sci. 47:139-147.

Spelman, R. J., and D. J. Garrick. 1998. Genetic and economic responses for within-family marker-assisted selection in dairy cattle breeding schemes. J. Dairy Sci. 81:2942-2950.

Spelman, R. J., D. J. Garrick, and J. A. M. van Arendonk. 1999. Utilisation of genetic variation by marker assisted selection in commercial dairy cattle populations. Livestock Prod. Sci. 59:51-60.

\section{APPENDIX 1}

\section{Superiority components of QAS over QAS}

Genetic response per year equals $\frac{i r \sigma_{g}}{L}$ where $i$ is selection intensity, $r$ is correlation between true and estimated breeding values, $\sigma_{g}$ is additive genetic standard deviation. In pathways where $i$ and $L$ are the same for QAS and QFS, superiority expressed as the difference in cumulative genetic response per year between QAS and QFS divided by QFS equals $\frac{r_{1} \sigma_{g 1}}{r_{2} \sigma_{g 2}}-1$, where subscripts 1 and 2 indicate QAS and QFS, respectively. But expressing $\sigma_{g}$ as $\sqrt{2 p q \alpha^{2}+\sigma_{g p}^{2}}$ (where $p$ is the frequency of the favorable QTL allele, $q=(1-p), \alpha$ is the average effect of gene substitution, and $\sigma_{g p}^{2}$ is the polygenic variance) leads to expression [3] in the text. 